I Comisión Nacional de Investigaciones Científicas y Estudios Técnicos (CONICET),

Buenos Aires, Argentina

juanpablogonnet@gmail.com

http://orcid.org/o0oo-0002-185I-9383

Juan Pablo Gonnet ${ }^{1}$

\title{
ORDEN SOCIAL, INTERACCIÓN Y SOCIEDAD EN LUHMANN. PERSPECTIVAS DE MÉTODO PARA LA INTEGRACIÓN DEL CONOCIMIENTO SOCIOLÓGICO
}

\section{INTRODUCCIÓN ${ }^{1}$}

Una de las principales contribuciones de la teoría de los sistemas sociales de Niklas Luhmann radica en la delimitación de un enfoque conceptual capaz de integrar, conectar y vincular la diversidad y complejidad de la investigación sociológica existente. En este sentido, la teoría presenta rendimientos que son altamente relevantes en el ámbito del método al otorgar recursos para la orientación, dirección y coordinación de la investigación social, propiciando cierto grado de coherencia en su desarrollo. ${ }^{2}$ Nos gustaría proponer que la valoración de este aporte no es menor en el contexto de una ciencia social cada vez más diferenciada y especializada, en la que los riesgos de desconexión, aislamiento y dispersión se han vuelto cada vez más evidentes. ${ }^{3}$

Los problemas asociados a la "división del trabajo" en la ciencia no son nuevos. Los mismos habían sido diagnosticados por Comte y Durkheim hace más de ioo años. Ambos entendían que este proceso estaba arraigado en la dinámica misma de la ciencia moderna. Sin embargo, mientras que para el primero este era un problema inherente a la división del trabajo científico, para el segundo, no era ese necesariamente el caso. El descubrimiento de problemas desconocidos y el trabajo sobre ellos es para Durkheim algo deseable, y hasta fundamental para el desarrollo científico. Si este proceso se transforma en crecimiento anárquico, desinterés y desintegración, esto se debe a que la división del trabajo no está generando los tipos de interdependencia sui generis 
que le son inherentes. Por esta razón, no debemos reprocharle directamente a la especialización la condición de dispersión. De hecho, el sociólogo francés rechaza aquellas soluciones que hacen de esta la causa del problema (Durkheim, 2008: 403 y ss). No se trata de ignorar las diferencias sino de reconocerlas e integrarlas. Durkheim considera que esto sólo es posible a través del desarrollo de "reglamentaciones" que, siendo sensibles a los resultados de las prácticas de investigación especializadas, puedan expresar de un modo lo suficientemente preciso el vínculo que guardan entre ellas. Así, no se debe buscar la restitución de una unidad perdida, sino la expresión de una existente. ${ }^{4}$ Una reglamentación adecuada sería aquella que pudiera coordinar las interdependencias entre las distintas especializaciones, de modo tal de que éstas puedan reconocer el horizonte en el que adquiere sentido su constitución.

No es otra la conclusión a la que llega Luhmann en relación a este problema que comenzó a ser gravitante en la sociología a partir de la segunda mitad del siglo XX. Desde sus primeras obras el teórico alemán muestra una preocupación permanente por la unidad de la disciplina (Luhmann, I973, I986, I994) y por la necesidad de desarrollar concepciones teóricas que puedan reflejar convincentemente la complejidad alcanzada en la comprensión del mundo social. Luhmann entiende que el riesgo de desintegración solo puede ser contenido a partir de un instrumental conceptual respetuoso de la complejidad, y no a través de la reposición de teorías o condiciones científicas previas. En conexión con esto, la teoría de los sistemas sociales busca elaborar un esquema lo suficientemente preciso, pero también flexible y abierto para el desarrollo y la especialización de la sociología, 5 que evite la dispersión, así como las unificaciones restrictivas.

En este trabajo buscamos sistematizar las prestaciones que esta teoría ofrece en materia de método para la integración de una división particular del trabajo sociológico que, de modo más o menos explícito, se ha vuelto dominante en la investigación social contemporánea. Nos referimos a aquella que se establece entre los análisis interaccionistas y los societales del orden social. Evidentemente, estos desarrollos han sido un aporte relevante para la sociología debido a que han puesto en evidencia una situación previa de "indistinción" entre interacción y sociedad. A pesar de ello, los rendimientos analíticos de esta maniobra se han vistos resentidos debido a que la distinción ha devenido en separación (lo micro y lo macro). Así, el tratamiento del problema del orden social se ha disuelto en perspectivas teóricas aisladas que se mantienen independizadas las unas de las otras. Esta situación ejemplifica, claramente, el problema que advertimos en los párrafos anteriores. La especialización deviene en dispersión cuando la atención a un campo lleva a desconocer las relaciones que este guarda con otro. Frente a este problema, una alternativa difundida ha sido la de la des-diferenciación. Esto es, interacción y sociedad son interpretadas como dimensiones de un continuo. Se podría decir, como dos 
momentos en la producción del orden social. Sin embargo, aquí no se articula la complejidad sino que se elimina. La especificidad y los límites de los órdenes sociales interactivos y societales tienden a desvanecerse.

En consecuencia, nos encontramos ante una distinción que es valiosa, que muestra el avance del conocimiento sociológico, pero cuyo desarrollo parece condenarnos o a una situación de desintegración o a la reposición de condiciones científicas previas. En este artículo deseamos mostrar de qué manera la teoría de los sistemas sociales presenta un enfoque conceptual idóneo para explotar heurísticamente la relación entre interacción y sociedad. Con este fin procedemos a una reconstrucción de los diversos modos en que la distinción es abordada por Luhmann y explicitamos las consecuencias metodológicas que de ellos se derivan.

\section{LA DISTINCIÓN DE NIVELES ANALÍTICOS}

Una de las primeras herramientas que nos brinda la teoría de los sistemas sociales para un tratamiento de los fenómenos interactivos y societales consiste en una delimitación precisa de niveles analíticos (Luhmann, I998a: 27 y ss.). En el nivel más alto de abstracción se posiciona la teoría general de sistemas, desde la cual se observa con pretensión de universalidad todo el espectro de posibles objetos de investigación que pudiesen asumir características sistémicas. Como se ocupa de mostrar Luhmann, en este primer nivel la teoría tiene un carácter eminentemente interdisciplinario y su desarrollo busca incorporar avances y discusiones que, perteneciendo a campos de estudio particulares, puedan tener consecuencias relevantes para la conceptualización de fenómenos sistémicos más amplios. Un ejemplo de esto es la idea de autopoiesis que, abstrayéndose de su directa referencia a procesos biológicos, pudo convertirse en un principio general para la descripción y caracterización del funcionamiento de sistemas complejos de diverso tipo (Luhmann, I990: 2).

Luego, en un segundo nivel analítico podemos distinguir tipos de sistemas cuyas diferencias no son relevantes o significativas para el primero. Aquí tenemos a los sistemas sociales, los psíquicos, las máquinas y los organismos. A la sociología le competen los primeros, pero esto no quita que pueda nutrirse del nivel más general de la teoría (re-especificándola). Lo que no es posible es la subordinación directa de los sistemas sociales a fenómenos biológicos o psíquicos y viceversa. Es decir, existe una irreductibilidad de los sistemas en este segundo nivel. Aquello que es afirmado para los sistemas vivos no puede ser trasladado automáticamente a los sistemas sociales o psíquicos. Si las propiedades sistémicas son comunes a todos los sistemas, deben registrarse en el primer nivel. Sin embargo, en este nivel se pueden abordar comparativamente a las equivalencias funcionales entre sistemas. Por ejemplo, cuando se destaca que el sentido es una dimensión constitutiva tanto de las operaciones psíquicas como de las sociales. 
En el tercer nivel de análisis ya tenemos la delimitación de sistemas de un mismo tipo. Es aquí en donde podemos distinguir, en el ámbito de los sistemas sociales, a la sociedad y a la interacción. Ciertamente, es posible identificar otros sistemas sociales, lo que muestra que esta enumeración no es exhaustiva y puede, eventualmente, ser ampliada. Por ejemplo, Luhmann (2010) encuentra que en la modernidad las organizaciones formales han llegado a constituirse en un tipo de sistema social específico y diferenciable tanto de la sociedad como de las interacciones. ${ }^{6}$ Adicionalmente, Luhmann sienta las bases para dar lugar a la conceptualización de otros sistemas sociales como, por ejemplo, los asociados a las dinámicas de protesta. Esto muestra que estamos ante un esquema que es evolutivo. Por último, los conflictos también constituyen un tipo particular de sistema social. A pesar de ello, al considerarlos como formaciones sistémicas "parasitarias" que se forman al interior de otros sistemas sociales (Luhmann, I998a: 35I), no se les otorga un lugar especial en este tercer nivel.

A pesar de su carácter elemental, este sencillo esquema de tres niveles resulta esclarecedor para el análisis sociológico. En el plano horizontal, se observa la irreductibilidad mutua de los distintos sistemas sociales. Luhmann reconoce que no existe un único sistema social, de hecho, habla de sistemas sociales. Esto significa que las interacciones, las organizaciones, las sociedades y las protestas son sistemas autónomos, con estructuras, dinámicas y comunicaciones distinguibles, es decir, no equiparables entre sí. En este movimiento, no sólo se destaca la particularidad de los sistemas, sino también su relacionalidad no jerárquica. Así por ejemplo, no es posible explicar a la sociedad a través de las interacciones, ni tampoco se puede concebir a estas últimas como epifenómenos de la sociedad. Ambas alternativas colocarían a una de las formaciones socio-sistémicas en un nivel analítico superior. Adicionalmente, tampoco resulta adecuado interpretar al vínculo entre interacción y sociedad de modo dialéctico. Dicha maniobra implicaría una reconducción de la teoría hacia una des-diferenciación de los sistemas al suponerse la posibilidad de una relación temporal entre ellos.

En el plano vertical del esquema se nos exige no confundir niveles. Por ejemplo, deducir del análisis de un sistema social particular conclusiones con respecto al plano general de los sistemas sociales. Esto ocurre cuando se subordina el problema del orden social al del orden societal, organizacional o interactivo, o cuando el fenómeno del conflicto se reduce a las contradicciones de clase. Es una tentación del análisis especializado olvidar el hecho de que existen otros sistemas que pueden operar tanto en su interior como en su entorno. De este modo, se puede reconocer que las interacciones se desarrollan en el marco de sistemas sociales más amplios como la sociedad y esta última aloja en su interior tanto sistemas interactivos como organizacionales. Por otra parte, tampoco es válido deducir del plano general de los sistemas sociales indicaciones con respecto a un sistema social particular sin una adecuada re-especificación. 
Las soluciones que deben dar las estructuras sistémicas a los problemas de orden en la interacción, las organizaciones y la sociedad son diferentes. No toda negación o desacuerdo que se produce en un encuentro presencial se transforma en un conflicto organizacional o societal - aunque ciertamente sea un conflicto social y, por ello, corresponda ser abordado por la teoría general de los sistemas sociales.

Por último, existe un riesgo más imperceptible del que nos previene la propuesta luhmanniana relacionado con obviar la consideración del nivel general de los sistemas sociales. En esta dirección, a la sociología podría bastarle con abordar distintos sistemas evitándose reflexionar en torno al problema de la unidad de los sistemas sociales. Por ejemplo, cuando se contrasta al orden de la interacción con el de los órdenes societales o macro-sociales, sólo se está sugiriendo que cada tipo de sistema procesa en sus propios términos el problema del orden, siendo irreductible la manera en que lo hace una interacción al modo en que lo hace una sociedad. Sin embargo, si se colocan a las distinciones entre diversos tipos de sistemas en el nivel de máxima generalidad del análisis sociológico, la teoría asume una forma dualista. Esta opción impide atender a la unidad de los sistemas sociales y como consecuencia, a sus relaciones e interdependencias. Luhmann, sin negar la autonomía de los sistemas sociales, no se contenta con sostener una dualidad. Lo que sucede es que la inquietud por la unidad no puede resolverse en el nivel de la diferenciación de los sistemas sociales, sino en el más general que los contiene.

Desde este lugar, queda rebatida la interpretación habitual que hace de la teoría de los sistemas sociales una perspectiva exclusivamente macrosociológica. Ciertamente, Luhmann tuvo un interés privilegiado en sus investigaciones por el sistema sociedad (particularmente, por la diferenciación funcional en la sociedad moderna), pero su perspectiva sociológica no absolutiza ni prioriza este objeto. La sociedad constituye un sistema social entre otros. De hecho, como mostraremos más adelante, un estudio completo de la sociedad como sistema omniabarcador requiere atender al funcionamiento de otros sistemas sociales en su interior. La teoría general de los sistemas sociales no es ni macro ni microsociológica.

Todo esto muestra que la pregunta por el orden social no puede responderse en el plano de la complejidad que se hace presente cuando contemplamos la diversidad de sistemas sociales. A pesar de ello, el análisis comparativo de cada uno de estos sistemas específicos puede brindarnos un mejor acceso para la comprensión de los sistemas sociales, a la vez que ayudarnos a controlar la transposición falaz de propiedades entre niveles. La teoría del orden social debe plantearse al nivel de la teoría general de los sistemas sociales, de modo tal de ser capaz de contemplar e integrar la contingencia de las formas en que este es posible; aprehendiendo del modo en que se viabiliza en cada uno de los sistemas particulares. Lo que emerge de este procesamiento es una teoría mu- 
cho más sensible a la complejidad del mundo social; complejidad que esta distinción de niveles logra preservar.

\section{DOBLE CONTINGENCIA: EL ORDEN SOCIAL MÁS ALLÁ DE LA DISTINCIÓN SOCIEDAD E INTERACCIÓN}

Un descubrimiento significativo de la teoría sociológica de posguerra estuvo relacionado con el reconocimiento de la interacción como un orden social sui generis.' Este hallazgo, lejos de haber dado lugar a una revisión y reconsideración de los fundamentos de la teoría del orden social, condujo mayoritariamente a la detección de déficits e insuficiencias de las perspectivas existentes. Así, en algunos casos, la teoría sociológica sucumbió a la interacción como el ámbito privilegiado desde el cual captar al orden social; en otros, se mantuvo una visión dualista que destacaba la inconmensurabilidad, irreductibilidad y hasta el antagonismo entre los órdenes societales y los interaccionales; y por último, propuestas más mesuradas, buscaron vincular dialéctica o agregativamente a estos dos ámbitos. Luego de un tiempo, parece ser bastante poco lo aprehendido de este estado de cosas. Siguiendo al esquema bosquejado en el apartado previo, podríamos decir que las dificultades para integrar el problema de la interacción se deben, al menos en parte, a la imposibilidad de distinguir satisfactoriamente niveles analíticos. Los debates se focalizaron en el tercer nivel (el de los distintos sistemas sociales) y evitaron dar lugar a una revisión del nivel superior (segundo nivel). Si la interacción y la sociedad son sistemas sociales, aquella teoría que los agrupe deberá ser consistente con ambas formaciones.

Luhmann (I976, I998a) encuentra en la teoría de la doble contingencia un punto de partida productivo para responder a esta exigencia. ${ }^{8}$ La doble contingencia describe una situación interactiva en la que dos interlocutores (alter y ego) reconocen mutuamente que disponen de alternativas de acción cuyo éxito depende de la acción del otro. ${ }^{9}$ Sin solución a este problema de indeterminación resulta inviable el orden social. Una novedad que aporta Luhmann tiene que ver con la necesidad de concebir a la doble contingencia como un problema permanente y no como una condición circunstancial y previa al orden. Desde este lugar, no existen soluciones definitivas y aseguradas de una vez y para siempre. El orden social debe lidiar constantemente con aquello que lo amenaza: con la complejidad social del mundo y con la complejidad del mundo social. No hay valores, contratos, dominación o procedimientos intersubjetivos que garanticen su vigencia. Toda propuesta de solución debe considerarse contingente en el contexto de la doble contingencia y, por tanto, como no necesaria y posible de otra manera.

Luhmann considera que en el marco de la doble contingencia cualquier acción o comunicación, intencional o no, tendrá valor informativo para los comportamientos individuales. En otros términos, cualquier suceso aporta determinación en el horizonte de la indeterminación. Es así como la contingencia 
posible se transforma en contingencia limitada a dos alternativas: aceptar lo sugerido o rechazarlo. El orden social es compatible con ambas. Puede continuar por el camino de la cooperación y la solidaridad o a través del conflicto y la negación. En ambos casos, de una manera u otra, se logra lidiar con el problema de la doble contingencia. El sistema social puede formar estructuras a partir de aquello que ha sido aceptado y modularlas de un modo normativo o cognitivo. Es decir, condenando las decepciones o aprendiendo de ellas. Es así como se entiende que el orden social no requiera de la reproducción de estructuras para su funcionamiento.

Ahora bien, ¿cómo se articula este desarrollo conceptual con la distinción interacción-sociedad? Atentos a nuestra hipótesis, habría que esperar que se cumplan con las precauciones de método elaboradas en el apartado anterior Si se pretende dar respuesta al problema general del orden social, la teoría de la doble contingencia deberá situarse en un nivel sistémico superior al de aquel en el que se encuentran los sistemas de interacción y societales. Esto significa que la teoría deberá poder contemplar las formas en que en cada uno de ellos es posible el orden, cuidando de no priorizar un sistema por sobre otro.

Para empezar, podemos entender a la diferenciación interacción-sociedad como dos formas de reducir la complejidad inscripta en las situaciones de doble contingencia. Uno debe atender a lo que siempre ha sido, a lo que se encuentra institucionalizado o es sabido por todos; pero también debe estar atento a los comportamientos y reacciones de aquellos que se encuentran en presencia física inmediata. Al ser sensible a la contingencia de las soluciones con respecto al problema del orden, la teoría de la doble contingencia permite contemplar tanto aquellas que son propias de los sistemas de sociedad, como aquellas que se viabilizan en los sistemas de interacción. La teoría nos obliga a no sucumbir ante las particularidades de un sistema social específico. La doble contingencia relativiza la preeminencia, tanto de la interacción como de la sociedad en la explicación del orden social.

Luego, resulta evidente cuan inadecuado es reducir el problema general del orden social a la realidad de la interacción. El hecho de que la doble contingencia sea presentada como una situación interactiva puede dar lugar a equívocos en este punto. Como mencionamos, la doble contingencia no debe ser entendida como una situación previa desde la cual debiera surgir el orden social; este hecho resulta empíricamente incomprobable. La doble contingencia no precisa presuponer nada acerca de lo previamente existente. No hay algo así como un estado de doble contingencia pura o de indeterminación total en la realidad social.

Por otra parte, si sostuviésemos que el orden social se genera en la interacción deberíamos asumir que este debe reconstituirse en cada momento, algo que negaría la existencia de la sociedad y sus estructuras. Hasta las reglas de la interacción tendrían que ser desconocidas. Más allá de esto, si aceptáse- 
mos a la interacción como un contexto en donde se "negocia" o se construye el orden, necesariamente deberíamos afirmar que esto sólo tiene sentido si dichos rendimientos sobreviven a la desaparición de la interacción. Es decir, habría que aceptar la distinción interacción/sociedad. De este modo, el teorema luhmanniano de la doble contingencia es antagónico con la subordinación del orden social a la interacción.

Por último, tampoco es aceptable la alternativa contraria. Esto es, responder al problema general del orden a partir de la formación de instituciones o sistemas societales. Los valores pueden ser útiles para mantener la integración en un partido político, pero no para la coordinación de una asamblea de miembros. La diferenciación de roles entre maestro y alumno puede ser significativa para la organización del sistema educativo, pero insuficiente para la organización de una clase. Si esto no fuese así, la doble contingencia dejaría de ser un problema permanente. El orden social estaría garantizado con anterioridad a toda interacción. Sin embargo, no estamos afirmando que las interacciones problematicen permanentemente a las estructuras sociales o al orden social. Lo que planteamos es que las interacciones tienen que resolver a su manera el problema del orden. Al mismo tiempo, a través de las interacciones la sociedad actualiza el problema de la doble contingencia y se digna a tratar con la contingencia de sus estructuras.

Es así como la teoría de la doble contingencia logra integrar en su tratamiento al problema del orden de la interacción y al de la sociedad en única perspectiva, al generalizar el problema del orden social. Desde este lugar, es inadecuada tanto la subordinación de un orden sistémico a otro, como el establecimiento de relaciones causales entre ellos. Ambas posibilidades nos llevan a desconocer la irreductibilidad mutua de los sistemas. Por otra parte, se logra superar la tesis de la dualidad de órdenes. El planteo de la doble contingencia muestra que interacción y sociedad se presuponen mutuamente, de manera que no resulta factible abstraer el análisis del orden de la interacción del de la sociedad y viceversa.

\section{LA DISTINCIÓN INTERACCIÓN-SOCIEDAD DESDE UNA PERSPECTIVA SISTEMÁTICA}

Dijimos que no todos los tipos de sistema social han estado presentes a lo largo de la historia. Por ejemplo, las organizaciones formales y los movimientos de protesta se vuelven preeminentes en la sociedad moderna, siendo excepcional su identificación en contextos sociales previos. Los sistemas de interacción y sociedad, en cambio, son constantes en la evolución social, a pesar de que sus características presenten importantes modificaciones a lo largo de ella. No hay formación social en la que no se pueda apreciar esta distinción sistémica y, por tanto, orden social que pueda interpretarse con independencia de ella. La tesis de la doble contingencia exhibe consistentemente esta inevitabilidad. 
Probablemente, la distinción entre sistemas de interacción y sociedad nunca haya sido totalmente elaborada en la tradición sociológica con anterioridad a la teoría de Luhmann. Al mismo tiempo, resulta evidente que la sociedad no es equiparable a una sumatoria de interacciones y estas, por su parte, presuponen una realidad social que las excede. Sin sistemas societales no hay interacción. Así y todo, gran parte de las comunicaciones societales se desarrollan en y a través de interacciones. El sistema de salud a través de las interacciones entre médico y paciente; el sistema económico por medio de operaciones de compra y venta en comercios; la educación a partir de la relación entre docente y alumno; y la política en sesiones parlamentarias. Es verdad que en la actualidad la sociedad cada vez más logra prescindir de las interacciones presenciales; a pesar de ello, aún no es claro si de lo que se trata es de la emergencia de nuevas modalidades de interacción (por ejemplo, no presenciales y tecnológicamente mediadas) o de una pérdida de protagonismo societal de la misma. ${ }^{\text {Io }}$ Sea como fuere, las sociedades constan de interacciones y por esta razón, se relacionan inevitablemente con ellas. Esto da lugar a problemas de coordinación que hacen de la distinción entre sistemas un hecho que no puede ser ni neutralizado ni reducido (Luhmann, I998a: 373).

Para Luhmann, la sociedad constituye el sistema social más amplio y abarcativo que incluye todo el campo de lo social. Es un sistema que no deja nada de lo social fuera suyo. Ninguna operación social existe más allá de los límites de la sociedad, por lo que nada de lo social puede resultarle ajeno. Es por esto que la sociedad no puede identificarse solamente a partir de sus estructuras. Inexorablemente incluye aquello que las decepciona o se aparta de ellas (Luhmann, 20I3: 20I). De esta manera, queda eliminada la posibilidad de entender a la sociedad, por ejemplo, a partir de un consenso normativo. Por el contrario, es en la disyunción entre el comportamiento conforme y el desviado en donde descansa su unidad.

la sociedad coloca a los individuos ante un esquema opcional. Ella concede como libertad lo que de ninguna manera puede cambiar: el que la comunicación pueda ser continuada a través del sí o del no, por medio de la conformidad o de la desviación (Luhmann, I998b: 64).

Gracias a esto, el sistema societal puede integrar a la historia en tanto que es su producto, pero también a la variación con respecto a ella y así a la evolución socio-cultural. La sociedad reproduce estructuras, pero también viabiliza sus transformaciones. El sistema garantiza su continuidad al contemplar en su horizonte todas las acciones y comunicaciones posibles y, por tanto, con sentido.

A diferencia de la sociedad, los sistemas de interacción presentan límites más claramente reconocibles. Estos se caracterizan por la presencia corporal inmediata de los interlocutores. Quien no está presente no pertenece al sistema y quien lo está tiene una consideración preferente, no necesariamen- 
te al interior del sistema, pero sí con respecto al entorno, es decir, a los que se encuentran ausentes. Las interacciones son sistemas que se generan por el tipo de acción recíproca que se establece cuando dos o más individuos se encuentran cara-a-cara y cuando, por esta razón, pueden percibir que son percibidos. Si se lee un libro se participa de la sociedad, pero no necesariamente de una interacción. Si el libro es leído en una biblioteca o en una sala de espera de un hospital, además se participará de un sistema interactivo. Las circunstancias combinadas de la presencia física en un tiempo y espacio determinado, y la alta significancia de los procesos de percepción mutua, hacen que en estos sistemas exista un exceso de información disponible (no es posible no comunicar) y una capacidad reducida de procesamiento. Por ejemplo, en una interacción solo se puede tratar un tema a la vez y no pueden hablar todos al mismo tiempo. Por estas características, estamos ante sistemas altamente expuestos a las perturbaciones (contingencias), razón por la cual se precisa de mecanismos específicos para la coordinación de las acciones en estas circunstancias.

En el marco de estas definiciones, es inevitable reconocer que estamos ante dos sistemas autónomos que poseen rendimientos sociales distintos. Esto se puede observar en el hecho de que un mismo acontecimiento puede constituirse como elemento de la sociedad y de la interacción; en otros términos, puede tener sentidos, consecuencias y horizontes temporales distintos. El otorgamiento de dinero a alguien que solicita ayuda en la vía pública es una acción que tiene relevancia tanto societal como interactiva. Coordina el comportamiento entre los presentes (responder a una demanda hecha personalmente) y, por medio del dinero, se coordina con otras interacciones (pasadas y futuras). Más allá de esta relevancia diferencial, ambos sistemas son fundamentales el uno para el otro. La interacción es un requisito para la sociedad. Podríamos aventurar que la sociedad depende del éxito de la interacción, aunque evidentemente, el fracaso de una interacción concreta no constituya una amenaza para las estructuras de la sociedad. Del mismo modo, la sociedad es la única que puede incrementar las posibilidades de éxito de la interacción, al poder asegurar vínculos y conexiones entre ellas. En palabras de Luhmann (I998a: 386):

Los sistemas de interacción continuamente pueden y tienen que ser abandonados y reiniciados. Esto exige una semántica que los trascienda, una cultura que conduzca este proceso en dirección a lo probable y lo acreditado. En este sentido, la sociedad actúa selectivamente sobre lo que existe como interacción, sin excluir por ello lo contradictorio y lo divergente. La selección social, por lo tanto, no determina; seduce por lo fácil y lo complaciente...

A pesar de esto, son las interacciones las que pueden habilitar, como dijimos, la evolución sociocultural, al permitir un campo de experimentaciones aprovechables. En relación con ello, resulta apropiado afirmar que la sociedad es resultado de las interacciones, aunque ella logre aquello que la interacción 
no puede: seleccionar interacciones que seleccionan la sociedad. En consecuencia, es posible sostener que sin diferencia con respecto a la interacción no habría sociedad, y sin diferencia con respecto a la sociedad no habría interacción.

El planteo de Luhmann es consistente con la distinción de órdenes sociales y con la necesidad de no subsumir uno a otro. Incluso la teoría de la sociedad, que sería aquella que trata el sistema que abarca el todo social, debe atender al hecho de que la forma en que define sus límites y reduce complejidad no es equivalente al modo en que lo hacen otros sistemas que operan bajo su égida. Mientras que el orden de la interacción depende de reglas, normas y procedimientos que son eficaces para la coordinación entre presentes, el orden de la sociedad descansa en la historia y la transformación de esas reglas. Dicho esto, la diferencia es elemental para el funcionamiento de los sistemas sociales, por lo que una teoría del orden social tiene que ser coherente con ambos lados.

La teoría general de sistemas formula únicamente unos conceptos muy abstractos y unas condiciones marco para el análisis de la realidad social. Aclara, por lo menos de manera fundamental, cómo se constituyen los sistemas sociales mediante procesos de autoselección y trazado de fronteras. Este proceso de construcción transcurre, sin embargo, bajo condiciones en cada caso peculiares, de tal manera que surgen tipos de sistema que no se dejan remitir los unos a los otros. No todos los sistemas sociales se forman de acuerdo a la fórmula interacción, ni todos según la fórmula sociedad [...]. Por consiguiente las teorías ordenadas a estos tipos de sistema tienen solo un alcance limitado. Ninguna aprehende al conjunto de la realidad social. Ni siquiera lo hace el sistema abarcador de la sociedad, que contiene en sí, ciertamente, los otros tipos de sistema, pero que no por ello es ya su prototipo (Luhmann, 2013: 203).

\section{LA DISTINCIÓN INTERACCIÓN-SOCIEDAD DESDE UNA PERSPECTIVA EVOLUTIVA}

Si bien destacamos que la distinción entre sociedad e interacción es constitutiva en la formación de los sistemas sociales, el grado en que se separan, diferencian, distancian o desacoplan se modifica a lo largo de la evolución. Mientras que en las sociedades primitivas o arcaicas lo característico es un acoplamiento fuerte entre estos sistemas, en las sociedades más complejas como la moderna se desarrollan acoplamientos más laxos. En tal sentido, la dimensión temporal incorpora una variable adicional al análisis de los vínculos entre los sistemas sociales. Las relaciones inter-sistémicas se modifican a lo largo de la evolución, por lo que no podemos dar por sentado el modo en que estas se realizan.

Antes de continuar, es preciso comprender que cuando hablamos de sistemas interactivos y societales no estamos denotando realidades mutuamente excluyentes o entidades separadas en el espacio o tiempo. Toda acción o comunicación que ocurre en una interacción también tiene lugar en la sociedad. En este sentido, una separación completa de los sistemas no resulta posible. Cuan- 
do hablamos de la distinción de sistemas, estamos refiriendo a perspectivas que definen límites operativos. La identidad sistémica no se recorta en un tipo de acción, sino a partir del sistema para el cual esa acción constituye un elemen-

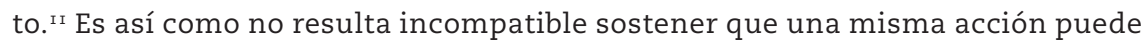
pertenecer simultáneamente a la sociedad y a la interacción en tanto da lugar a relacionalidades distintas. Lo significativo es que en la interacción se cristaliza esta diferencia. En una interacción concreta se hace manifiesto el sistema de la sociedad y el de la interacción. En consecuencia, cuando hablamos de la diferenciación entre interacción y sociedad no debemos entenderla como la separación de algo que estaba unido, sino que referimos al cambio en las relaciones entre dos sistemas que operan simultánea y autónomamente.

Luhmann (2013) considera que, en las formaciones sociales arcaicas, la sociedad se halla constituida más cercanamente a la interacción. En estas, las estructuras sociales definen y regulan de un modo altamente preciso a las interacciones entre sus miembros, por ejemplo, a través de pautas rituales detalladas o de la subordinación completa del individuo a su posición social. La interacción no pierde autonomía, no deja de ser un sistema, pero sus estructuras se encuentran fuertemente acopladas a las de la sociedad. Por otra parte, la sociedad es más sensible a lo que acontece en los encuentros sociales presenciales. Cualquier desviación debe ser rápidamente reprimida o controlada. En este contexto, las interdependencias entre los sistemas son estrechas, algo que es propio de sociedades poco complejas y con menos capacidad de procesar la contingencia social. Interacción y sociedad se limitan mutuamente.

A medida que el orden social se va complejizando (por ejemplo, con la formación de grandes centros urbanos, con la división del trabajo y el desarrollo del individualismo), la sociedad ya no puede regular a las interacciones tan de cerca, por lo que debe hacer reflexiva su diferencia con respecto a ellas. Si el sistema societal pretende coordinar sus acciones tiene que, necesariamente, separarse más contundentemente de la realidad de la interacción. Así, muchas de las contingencias y desviaciones de las situaciones cara-a-cara no tendrán mayores consecuencias para el funcionamiento de la sociedad. Sin embargo, en tanto que la sociedad se reproduce a través de las interacciones, esta garantiza condiciones mínimas para su desarrollo. Por ejemplo, a través de los medios de comunicación simbólicamente generalizados, la diferenciación de roles o la disciplina organizacional. En este movimiento, las interacciones a la vez que se hacen más dependientes de estas condiciones generalizadas, también se vuelven más independientes debido a que obtienen más grados de libertad en relación con la sociedad. Esto explica la relevancia que adquieren las normas de la interacción en las sociedades modernas. ${ }^{\mathrm{I} 2}$ La interacción ya no necesita ser sociedad (Luhmann, 2013: 205); debido a esto se desarrollan reglas, mecanismos y estructuras específicas para lidiar con la complejidad de las situaciones cara-a-cara. ${ }^{13}$ Bajo esta circunstancia es de esperar que los problemas de 
coordinación presencial no devengan automáticamente en problemas societales, del mismo modo que los conflictos societales puede que no afecten a las interacciones. De manera que tenemos una mayor complejidad producto del distanciamiento creciente entre sistemas. ${ }^{\mathrm{I4}}$

Veamos algunos de los riesgos de los que nos previene este diagnóstico evolutivo. En primer lugar, al hacer comprensible la razón por la que el descubrimiento del orden de la interacción ha sido posible en sociedades altamente complejas como la nuestra (en donde la autonomía de los sistemas de interacción se ha vuelto evidente), nos previene de convertir esta notoriedad social en privilegio analítico. Los problemas de coordinación interactiva no sustituyen a los de coordinación societal, a pesar de que estos se vean cada vez más limitados para explicar a los primeros. En segundo lugar, al ampliarse la brecha entre interacción y sociedad, los rastros de esta última serán más difusos en las interacciones concretas. Para ser claros y evitar equívocos: la coordinación del sistema sociedad se realiza en las interacciones, sin embargo, esto no significa que el orden del sistema interactivo (de mutua presencia) se encuentre resguardado por las estructuras de la sociedad. Por último, la diferenciación evolutiva entre sistemas hace totalmente viable que el desorden en uno de ellos no resulte contradictorio con el orden del otro (Luhmann, I987: I26). Así, la inestabilidad que podemos observar en las interacciones no implica, necesariamente, desorganización de la sociedad, y esta última no deviene en desorden interactivo.

\section{CONSECUENCIAS DE MÉTODO PARA EL ANÁLISIS SOCIOLÓGICO}

A lo largo del trabajo expusimos los vínculos y relaciones que se establecen entre interacción, sociedad y orden social, y pusimos en evidencia ciertas exigencias metodológicas que se desprendían de ellos. En este apartado, sistematizamos, integramos y resumimos estos aportes. Proponemos organizar la exposición desagregando de a pares los vínculos que se establecen entre los distintos niveles y tipos de formación socio-sistémica. A continuación abordamos las implicancias que la consideración de cada uno de estos ámbitos (interacción, sociedad y orden social) acarrea para el tratamiento de cada uno de los otros. En la siguiente tabla exponemos las seis posibilidades que quedan perfiladas.

\begin{tabular}{l|c|c|c}
\hline & Interacción & Sociedad & Orden Social \\
\hline Interacción & $\mathrm{X}$ & $\mathrm{A}$ & $\mathrm{B}$ \\
\hline Sociedad & $\mathrm{C}$ & $\mathrm{X}$ & $\mathrm{D}$ \\
\hline Orden Social & $\mathrm{E}$ & $\mathrm{F}$ & $\mathrm{X}$ \\
\hline
\end{tabular}




\section{A) Implicancias de la interacción para el análisis de la sociedad}

Las interacciones inevitablemente traen aparejadas consecuencias para el análisis de la sociedad al ser sistemas que funcionan en su interior. Los eventos interactivos son los elementos de los que consta la sociedad, por lo que es en las interacciones en donde se reproducen sus estructuras. No obstante, este proceso no puede ser dado por supuesto en razón de la autonomía y las particularidades propias de los sistemas de interacción. Al ser sistemas diferenciados de la sociedad, las interacciones pueden dar lugar a resistencias y hasta poner límites a esas estructuras. Este es el caso, por ejemplo, de los "ajustes secundarios" en instituciones totales (Goffman, 200I: I90 y ss.) o de las redes informales en las organizaciones. Adicionalmente, en determinadas circunstancias, las interacciones pueden estimular procesos evolutivos y de cambio estructural (Münch, I987). Después de todo, la doble contingencia se regenera en cada interacción. La precaución metodológica que emerge de estas distintas posibilidades consiste en evitar que el problema de la interacción sea obturado por el análisis societal. Cuando el funcionamiento de la sociedad se explica, meramente, en términos de hegemonía, integración cultural o estructuras de diferenciación funcional, lo que se está negando es la existencia de otras formaciones sistémicas que operan en su interior. En este sentido, sería adecuado sostener, por ejemplo, que el fenómeno de la diferenciación funcional no agota la conceptualización de la sociedad (Nassehi, 20 I I). Estas premisas podrían contribuir a que los análisis de la sociedad se hagan más permeables a los numerosos descubrimientos que se han hecho en el ámbito de la interacción. De igual modo, esto podría convertirse en un estímulo para la actualización y complejización de la teoría de la sociedad.

\section{B) Implicancias de la interacción para el análisis del orden social}

La interacción se configura como la situación elemental de la que inevitablemente parte el análisis del orden social, aunque esto no signifique que ella tenga un privilegio especial en su explicación. Las interacciones presenciales configuran sistemas, pero también son eventos que pertenecen y reproducen a otros sistemas. Ahora bien, el análisis del orden social no puede desconocer que en las interacciones se presentan situaciones de doble contingencia que deben ser resueltas, por más que reconozcamos que nunca se parte de situaciones totalmente indeterminadas (doble contingencia pura). Como vimos más arriba, la evolución socio-cultural depende del reconocimiento de este hecho. El análisis de la interacción nos recuerda que el problema del orden social es un problema permanente. Es así como las interacciones no pueden ser concebidas como epifenómenos del orden social, o como su mera consecuencia. En tal dirección, es fundamental atender al modo en que se resuelve el problema del orden en estas circunstancias. En los encuentros presenciales los interlocutores pueden remitirse a lo existente, a lo previamente disponible, pueden 
proponer algo nuevo o utilizar cualquier casualidad como forma de auto-determinación.

\section{C) Implicancias de la sociedad para el análisis de la interacción}

Una de las inquietudes que dio lugar al presente ensayo remite a la creciente valoración o interés que vienen despertando los fenómenos interaccionales en la sociología y en otras ciencias sociales. Sin embargo, una de las principales limitaciones de estas indagaciones tiene que ver, justamente, con el olvido del carácter interactivo de los procesos observados. Estas investigaciones tienden a omitir la referencia sistémica de sus descubrimientos, lo que como vimos genera reduccionismos al mismo tiempo que generalizaciones poco controladas. Se toman como cuestiones de teoría general procesos que tienen una naturaleza estrictamente interaccional. Error en el que caen también aquellos que cuestionan los limitados alcances societales de los fenómenos y/o dinámicas interactivas. En este sentido, es fundamental distinguir sistemas de referencia. Por otra parte, resulta necesario asumir que las interacciones presuponen a la sociedad. Esta existe antes y después de que estas desaparezcan. Desde este lugar, es la sociedad la que viabiliza a las interacciones. Aporta recursos, medios, roles y expectativas que son valiosas, al menos para la iniciación de los encuentros sociales. Y aunque estos sean insuficientes para resolver las complejidades que se presentan en la realidad de la interacción, no dejan de ser relevantes. De hecho, si fueran suficientes se sabotearía la misma distinción entre sociedad e interacción. Finalmente, cabe mencionar que el grado de complejidad alcanzado por el sistema sociedad es el que delimita el tipo de interdependencias que este guarda con la interacción. La diferenciación de la interacción con respecto a la sociedad es producto de la evolución. En cualquier caso, esto no significa que la interacción pierda relevancia social o que su operar sistémico carezca de consecuencias que deban ser atendidas y reconocidas.

\section{D) Implicancias de la sociedad para el análisis del orden social}

Reconocer a la sociedad como sistema impide concebir al orden social como producto de procedimientos y/o mecanismos interactivos. Por ejemplo, entenderlo como resultado de intercambios, negociaciones, justificaciones pragmáticas, contratos, acuerdos de trabajo, etnométodos, relaciones de reciprocidad o como un hecho espontáneo. A pesar de que todo análisis general del orden social parta de la ponderación abstracta de una situación interaccional15 (doble contingencia), no es posible demostrar ni empírica ni fenomenológicamente la ausencia de un orden previo (o concomitante) a la misma. En otras palabras, que no exista sociedad. Hablar de sociedad implica evitar el equívoco de concebir que el orden social sucede al caos o a la lucha de todos contra todos. Aquí se hace justicia a la observación durkheimiana en torno a las dimensiones sociales previas que viabilizan cualquier proceso interactivo ("lo no contractu- 
al del contrato"). Sin embargo, lo anterior no contradice el reconocimiento de la autonomía del orden de la interacción. El orden de la sociedad ni lo explica ni lo garantiza. Por consiguiente, tampoco la explicación de este es suficiente para dar acabadamente cuenta del fenómeno general del orden social.

\section{E) Implicancias del orden social para el análisis de la interacción}

Como mencionamos, el problema del orden social no puede restringirse al tratamiento del problema del orden de la interacción ya que esto significaría comprenderlo como un logro situado o situacional que debe hacerse o rehacerse cada vez que se encuentran dos o más personas. Siendo la interacción un tipo de sistema específico, la teoría del orden social deberá tener en cuenta el modo en que es posible la coordinación de las acciones en estas circunstancias, pero no deberá hacer de ello el fundamento general del orden social. Lo que implica relativizar los alcances sociales de los procedimientos, reglas, recursos y dispositivos que se actualizan en estos sistemas. Por ejemplo, los "acuerdos de trabajo" que se producen en el marco de una movilización colectiva no pueden ser generalizados como explicaciones suficientes para una teoría del orden social. Del mismo modo, un conflicto que desata la disolución de una asamblea universitaria no puede ser tomado como una crisis del orden social. Esta observación también valdría para el caso de los famosos "experimentos de ruptura" (Garfinkel, 2006). Es así como el sistema de interacción deberá ser el marco específico desde el cual se tornen comprensibles aquellos mecanismos organizadores de estos contextos presenciales. Más allá de estos cuidados, los análisis de la interacción pueden aportar a la teoría general del orden social. De hecho, han sido sumamente relevantes para cuestionar a las ampliamente difundidas tesis de la integración cultural (Archer, I996).

\section{F) Implicancias del orden social para el análisis de la sociedad}

En este último caso, se trata de no permitir que el carácter problemático del orden social (doble contingencia) sea desactivado por el análisis de la sociedad. Dijimos que esto es algo fundamental para explicar su dinamismo y evolución. Ahora bien, esto no debe llevarnos a afirmar que las estructuras societales se encuentren en permanente transformación. Si tal fuera el caso, el orden social sería un fenómeno sumamente inestable. Aunque es factible constatar la existencia de variaciones permanentes en la sociedad, no todas ellas son igualmente atendidas, y mucho menos retomadas en eventos o comunicaciones subsiguientes. Sea como fuere, el análisis de la sociedad debe atender a este hecho porque es constitutivo de ella. Adicionalmente, existen otros sistemas sociales que se organizan y ordenan de forma autónoma. Es así como la pregunta por el cómo es posible la sociedad no puede ser equivalente a la pregunta por el cómo es posible el orden social. El orden de la sociedad no agota el espectro completo del fenómeno del orden social y el modo en que este es viable. Es 
decir, no constituye el nivel de máxima generalidad del análisis sociológico. Un gesto amable o una señal de cortesía pueden ser suficientes para organizar un encuentro social, pero claramente, no la sociedad. Con todo, estos ordenamientos son parte de ella y pueden tener consecuencias más o menos relevantes en sus dinámicas. Como, por ejemplo, cuando las reglas o las normas de la interacción facilitan la reproducción de estructuras que son contradictorias con las de la sociedad o cuando, a la inversa, las protegen y reafirman.

\section{A MODO DE CIERRE}

Al comienzo del trabajo planteamos el problema de la dispersión del conocimiento sociológico agudizada por la creación y delimitación casi constante de nuevos campos de investigación. En conexión con este punto, sostuvimos que el problema no debía atribuirse a la especialización en sí misma, la cual constituye una tendencia inevitable del desarrollo de la ciencia y, por tanto, también de las ciencias sociales. Desde nuestra perspectiva, el carácter objetable se encuentra en el hecho de que esta dinámica se desenvuelve de modos teórica y metodológicamente poco controlados, afectándose el potencial de diálogo e irritación recíproca entre campos y áreas de investigación. En este artículo afirmamos que esta situación se expresa paradigmáticamente en la disociación entre los análisis interactivos y societales del orden social; entre las perspectivas macro y micro sociológicas. Frente a esto, recuperamos un conjunto de distinciones que nos brinda la teoría general de los sistemas sociales para el abordaje de los vínculos entre interacción y sociedad. A partir de ellas, procuramos elaborar algunas indicaciones de método para el análisis general del orden social y el análisis específico del orden en esos ámbitos. A continuación resumimos estos aportes.

En primer lugar, recuperamos la distinción de niveles analíticos propuesta por Luhmann. Aquí pudimos reconocer que el abordaje de los sistemas sociales se sitúa en un plano de mayor generalidad y abstracción que el de los sistemas sociales específicos (interacción, sociedad, organización y sistemas de protesta). En este marco, no sólo resulta inadecuado asumir la preeminencia analítica de uno de estos sistemas en la explicación general del orden social, sino que también es incorrecto asumir esta preeminencia en el análisis particular de un sistema específico. En un caso, la dificultad es la extrapolación falaz y en el otro, la omisión de interdependencias sistémicas.

En segundo lugar, vimos que la teoría de la doble contingencia, como respuesta general al problema del orden social, contempla en su tratamiento tanto la conformación sistémica interactiva como la societal, sin priorizar a ninguna de ellas. Al no proponerse como una explicación genética, el planteo no se deja subsumir a una resolución interactiva. Pero al hacer de la doble contingencia un problema permanente y actualizable en cada interacción, habilita a contemplar al modo en que el mismo es solucionado en estas instancias. 
En tal sentido, se hizo evidente que el fenómeno del orden social atañe a la sociedad y a la interacción por igual. Podríamos decir, a la coordinación presencial y a la coordinación entre interacciones.

Por último, la distinción sistemática y evolutiva entre interacción y sociedad nos permitió mostrar que estamos ante dos formas elementales de reducción de complejidad en el sistema social y, por tanto, ante dos formas de orden distintas. Los sistemas de interacción emergen cuando dos o más personas se encuentran en presencia corporal inmediata, y los societales son aquellos que contemplan a todo el campo de ocurrencias sociales. Desde esta clarificación se vuelven evidentes las interdependencias que se establecen entre ellos. Los sistemas de interacción pertenecen al sistema sociedad y este se reproduce a través de eventos interactivos. Por consiguiente, constatamos que no resultan aceptables ni la des-diferenciación, ni la dualidad.

Para culminar, nos gustaría justificar por qué razón creemos que estas consideraciones de método podrían ser relevantes para las ciencias sociales en América Latina. Ciertamente, la desconexión del conocimiento sociológico atenta contra la construcción de una mirada lo suficientemente compleja y general de lo social y de la sociedad en todas partes, pero en la periferia este hecho acarrea serias dificultades para comprender el modo en que se producen, estabilizan y reproducen las desigualdades regionales que nos afectan y constituyen. La dispersión de la investigación social de la que hablamos no es privativa de las ciencias sociales de nuestra región, sin embargo, es probable que las consecuencias de tal estado de cosas sean más críticas en nuestras latitudes. En consonancia con esto, los aportes metodológicos presentados pueden resultar pertinentes.

Recibido en 6/8/20I9 | Revisado en 26/12/2019 | Aprobado en 2/2/2020

Juan Pablo Gonnet es doctor en ciencias sociales por la Universidad de Buenos Aires, investigador del CONICET (Comisión Nacional de Investigaciones Científicas y Estudios Técnicos) y profesor de teoría sociológica en la Universidad Nacional de Córdoba. Áreas de interés: teoría de los sistemas sociales, teorías del orden social y sociología de la moral. Publicaciones recientes: "Orden social y conflicto en la teoría de los sistemas de Niklas Luhmann" (Cinta de Moebio Revista de Epistemología de las Ciencias Sociales, 6I) y "La doble contingencia como clave para la redefinición del concepto de orden social" (Estudios Sociológicos, 36/ı06). 


\section{NOTAS}

I Quisiera agradecer a los comentarios que Sergio Pignuoli Ocampo hizo a una versión preliminar de este escrito.

2 En este trabajo partimos del supuesto de que resulta inadecuado asumir la distinción teoría/método en términos dualistas, es decir, como ámbitos autonomizados y escindidos en la lógica de la investigación social. Acerca de este punto, véase Pignuoli Ocampo (2016). Por otra parte, en relación con esta distinción, nos interesa explotar la "re-entry" de la teoría en el método.

3 En la actualidad, la especialización adquiere un carácter auto-propulsado. Cada vez son más frecuentes las publicaciones que inauguran (o pretenden inaugurar) nuevos campos de indagación.

4 "Para que la ciencia sea una, no es necesario que se mantenga entera en el campo visual de una sólo y única conciencia - lo cual, por otra parte, es imposible: basta con que todos los que la cultivan sientan que colaboran en una misma obra" (Durkheim, 2008: 408).

5 Luhmann (1994: I95) habla de "producción de inseguridad estructurada".

6 Esta hipótesis no es definitiva. Luhmann (2013) también considera que las organizaciones existieron en otros momentos históricos, siendo su preeminencia o grado de diferenciación aquello que resulta variable.

7 En este punto, vale hacer una aclaración. Las realidades interactivas venían siendo materia de estudio sociológico desde Simmel en adelante. Por ejemplo, se pueden reconocer referencias a la interacción en las obras de Schutz, Mead, el interaccionismo simbólico y hasta el mismo Parsons. Sin embargo, es su distinción sistémica la que se consolida en este momento, disputándose así el presupuesto de continuidad o superposición analítica entre interacción y sociedad. En este sentido, podemos identificar a las obras de Goffman (I99I) y Garfinkel (2002) como fundacionales en lo que respecta a esta tesis. Sobre este tema, véase Rawls (2009). Lo que nos interesa plantear es que en este momento se abre la posibilidad de que la teoría sociológica mantenga una perspectiva dual en relación al problema del orden social. Pasamos de la superposición a la desvinculación. 
8 Para nuestros propósitos resulta significativo que Luhmann (2002: 330) inscriba el planteamiento de este problema en el contexto histórico de desarrollo de la teoría sociológica: "La teoría de la doble contingencia debe ser pensada en un orden que sirve para otro tipo de explicación, es decir, para dar cuenta de cómo es posible el orden social: ¿cómo se puede romper con la circularidad inherente de la doble contingencia? Este tipo de preguntas, que siguen el estilo inaugurado por Kant sobre las condiciones de posibilidad, no son preguntas que puedan responderse con pruebas empíricas de tipo histórico. Surgen más bien, y esto puede comprobarse en una sociología del conocimiento, para que en épocas de transición sirvan de estímulo heurístico con el fin de que se busque una respuesta plausible en lo referente a la complejidad del orden social".

9 En este espacio no nos ocuparemos de desarrollar acabadamente la temática de la doble contingencia, cuestión que hemos tratado en otro lugar (Gonnet, 20I5, 2017). También existen otros artículos que han abordado en profundidad la cuestión (Pignuoli Ocampo, 2013; Vandearstraeten, 2002).

Io Retomamos este punto en el próximo apartado.

I I "Puesto que en gran medida (no exclusivamente) la comunicación se realiza como interacción, sería un error pensar en una diferencia sistema/entorno, o aun suponer que la sociedad consta de operaciones abstractas y la interacción, en cambio de operaciones concretas (comunicación, acciones). La sociedad no excluye, incluye la interacción. Por lo tanto, no se da una separación entre distintos tipos de acción: sociales e interaccionales. La diferencia más bien estructura el campo no diferenciado de las operaciones elementales. La abstracción se vuelve entonces, en gran parte, relevante para la interacción en la interacción, pero no se deriva de la misma, sino de su sociabilidad y por ello no está disponible localmente" (Luhmann, I998a: 377).

I2 En esta dirección, Luhmann (I987: II7) sostiene: “...the society and its primary functional subsystemas will become less dependent upon rules of interaction and will require an understanding of their own structural condi- 
tions. On the other hand, interactions will also become more dependent on their own autopoietic self-realization, particularly on something that can be summarized as 'taking the role of the other' (Mead) or as adapting to 'double contingency' (Parsons)".

I3 Goffman (I967) habla de la "moral de la interacción" y del hecho de que en nuestra sociedad se vuelve cada vez más inadecuado el invocar el rango, el status o la clase en las situaciones interactivas (Goffman, I95I).

I4 Adicionalmente, cabe señalar que el desarrollo de técnicas de comunicación como la escritura y la imprenta, al alejar la acción y la comunicación de la temporalidad y espacialidad limitada de las situaciones presenciales, posibilitan una separación mayor entre interacción y sociedad (Luhmann, I987: II6).

I5 Además de Luhmann, esto es común a Simmel, Schutz, Mead, Parsons, Blummer, Elster y Garfinkel, entre otros.

\section{REFERENCIAS BIBLIOGRÁFICAS}

Archer, Margaret. (I996). Culture and agency. The place of culture in social theory. Cambridge: Cambridge University Press.

Durkheim, Émile. (2008). La división del trabajo social. Buenos Aires: Gorla.

Garfinkel, Harold. (2006). Estudios en etnometodología. Madrid: Anthropos.

Garfinkel, Harold. (2002). Ethnomethodology's program. Maryland: Rowman and Littlefield Publishers.

Goffman, Erving. (200I). Internados. Buenos Aires: Amorrortu editores.

Goffman, Erving. (I99I). El orden de la interacción. In: Winkin, Yves (ed.). Los momentos y sus hombres. Barcelona: Paidós, p. I69-205.

Goffman, Erving. (I967). Interaction ritual. Essays on faceto-face behavior. London: Penguin Books.

Goffman, Erving. (I95I). Symbols of class status. The British Journal of Sociology, 2/4, p. 294-304. 
Gonnet, Juan Pablo. (20I7). La doble contingencia como clave para una redefinición del concepto de orden social. Estudios Sociológicos, 36/ı06, p. 47-72.

Gonnet, Juan Pablo. (20I5). Las dos representaciones del problema del orden social en la teoría sociológica de Niklas Luhmann. Athenea: revista de pensamiento e investigación social, I5/I, p. 249-269. Disponible en <http://dx.doi. org/I0.5565/rev/athenea.I480>.

Luhmann, Niklas. (2013). Interacción, organización, sociedad. Aplicaciones de la teoría de sistemas. In: La moral de la sociedad. Madrid: Editorial Trotta, p. I97-2I3.

Luhmann, Niklas. (2010). Organización y decisión. Ciudad de México: Herder.

Luhmann, Niklas. (2002). Introducción a la teoría de los sistemas. Madrid: Editorial Anthropos.

Luhmann, Niklas. (1998a). Sistemas sociales. Lineamientos para una teoría general. Madrid: Editorial Anthropos.

Luhmann, Niklas. (I998b). El concepto de sociedad. In: Complejidad y modernidad. De la unidad a la diferencia. Madrid: Editorial Trotta.

Luhmann, Niklas. (I994). Los problemas de la investigación en sociología. Convergencia. Revista de Ciencias Sociales, 7, p. I94-I98.

Luhmann, Niklas. (1990). The autpoiesis of social systems. In: Essays on self-reference. New York: Columbia University Press, p. I-2I.

Luhmann, Niklas. (I987). The evolutionary differentiation between society and interaction. In: Alexander, Jeffrey et al. (eds.) The micro-macro link. Los Angeles: University of California Press, p. 319-336.

Luhmann, Niklas. (1986). La teoría moderna del sistema como forma de análisis social complejo. Revista Sociológica, I/I, p. 7-24.

Luhmann, Niklas. (1976). Generalized media and the problem of contingency. In: Explorations in general theory of social science. Essays in honor of Talcott Parsons. New York: The Free Press, p. 507-532.

Luhmann, Niklas. (I973). La sociología como teoría de sistemas sociales. In: Ilustración sociológica y otros ensayos. Buenos Aires: Editorial Sur. 
Münch, Richard. (1987). The interpenetration of microinteraction and macrostructures in a complex and contingent institutional order. In: Alexander, Jeffrey et al. (eds). The micro-macro link. Los Angeles: University of California Press, p. 319-336.

Nassehi, Armin. (20II). La teoría de la diferenciación funcional en el horizonte de sus críticas. MAD, 24, p. I-29.

Pignuoli Ocampo, Sergio. (2016). Reflexiones sobre la distinción entre teoría y método desde una perspectiva sistemática. In: Torres Rivas, Edelberto \& Gonnet, Juan Pablo. (eds.). La teoría social en América Latina. Problemas, tendencias y desafíos actuales. Buenos Aires: CONICET.

Pignuoli Ocampo, Sergio. (2013). Doble contingencia y orden social desde la teoría de los sistemas de Niklas Luhmann. Sociológica, 28, p. 7-40.

Rawls, Anne. (2009). An essay on two conceptions of social order. Journal of Classical Sociology, 9/4, p.500-520.

Vandearstraten, Raf. (2002). Parsons, Luhmann and the theorem of double contingency. Journal of Classical Sociology, 2, p. 77-92. 


\section{ORDEM SOCIAL, INTERAÇÃO E SOCIEDADE EM LUHMANN. PERSPECTIVAS DO MÉTODO PARA A INTEGRAÇÃO DO CONHECIMENTO SOCIOLÓGICO}

\section{Resumo}

As análises da interação foram fundamentais para discutir alguns dos pressupostos mais consolidados da reflexão sociológica sobre a ordem social e seu funcionamento. No entanto, essa complexidade foi dissolvida na identificação de uma dualidade, e mesmo oposição, entre a ordem da interação e aquela da sociedade. É assim que as pesquisas dessas áreas sociais tendem a permanecer dissociadas, separadas e desconectadas. Nesse movimento, não apenas a possibilidade de alcançar um tratamento unificado do problema da ordem social está bloqueada, mas, ainda pior, inevitavelmente se opera com visões reducionistas. O objetivo desse artigo é reconstruir as contribuições que a teoria geral dos sistemas sociais oferece em termos de método para evitar esses riscos analíticos.

\section{SOCIAL ORDER, INTERACTION AND SOCIETY IN LUHMANN. METHODOLOGICAL APPROACHES TO THE INTEGRATION OF SOCIOLOGICAL KNOWLEDGE}

\footnotetext{
Abstract

Analyses of interaction have been fundamental to debating some of the most consolidated assumptions of sociological reflection on social order. However, this complexity has been dissolved in the identification of a duality, and even an opposition, between the order of interaction and that of society. Consequently, inquiries into these social areas tend to remain dissociated, separated and disconnected. In the process, not only has the possibility of achieving a unified treatment of the problem of social order been blocked, but even worse, reductionist perspectives have inevitably taken hold. The purpose of this article is to reconstruct the methodological contributions that the general theory of social systems offers to avoid these analytical risks.
}

\section{Palavras-chave}

Interação; método; ordem social; sociedade; teoria geral dos sistemas sociais.

Keywords

Interaction; method; society; social order; general theory of social systems. 